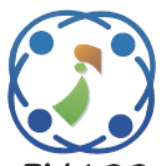

\title{
Design of Minkowski Fractal Iteration in Monopole Patch Antenna
}

\author{
Bharathraj Kumar Medhal ${ }^{1 *}$ \\ Preveen Jayappa ${ }^{2}$ \\ Jagadeesha Shivamurthy ${ }^{3}$ \\ ${ }^{1}$ Department of Electronics and Communication Engineering, \\ Shri Dharmasthala Manjunstheshwara Institute of Technology, Ujire, India \\ ${ }^{2}$ Department of Electronics and Communication Engineering, \\ Alva's Institute of Engineering \& Technology, Moodbidri, India \\ ${ }^{3}$ Department of Electronics and Communication Engineering, \\ Sri Venkateshwara Collage of Engineering and Technology, India \\ * Corresponding author's Email: bharathrajkumar100@gmail.com
}

\begin{abstract}
Many communication applications the antenna with smaller size, less weight and high efficiency is in demand. Monopole antenna able to process the wideband application and light weight. However, monopole antenna has the low return loss that affects the efficiency of the antenna. Fractal shapes has been applied in the monopole antenna to increase the return loss and efficiency. In this research, the Microstrip Minkowski Patch Antenna (MMPA) is proposed to increase the efficiency of monopole antenna. Minkowski Iteration Fractal antenna technique is applied to optimize the design in iterative manner for bandwidth and frequency tuning for wideband applications. The experimental evaluation shows that the proposed MMPA method has the higher bandwidth and more stable gain compared to the other fractal antennas. The return loss of the proposed MMPA's second iteration is $-21.38 \mathrm{~dB}$ at 3.2 $\mathrm{GHz}$ and base design has $-30.37 \mathrm{~dB}$ at $3.6 \mathrm{GHz}$. Additionally, the MMPA is compared with existing Koch fractal antenna in terms of patch dimension and return loss. The patch dimension of the MMPA is $25 \times 20 \mathrm{~mm}^{\wedge} 2$, it is less when compared to the Koch fractal antenna.
\end{abstract}

Keywords: Fractal antenna, Microstripminkowski patch antenna, Minkowski iteration fractal, Monopole antenna and wideband application.

\section{Introduction}

The tiny multiband antenna receives huge attention in the wireless communication systems due to the modern communication systems and its growing application areas. The important requirements of the modern tiny antennas are less weight, higher performance and small size. Moreover, the microstrip antennas are considered as one of the miniaturized antennas with various benefits such as inexpensive, less cost, conformability and so on. The design is easy, while considering the simple radiating patch shapes [1]. In miniaturized antennas, monopole antennas are considered as preliminary aspect that is broadly utilized in mobile terminal devices. The monopole antenna is integrated in the mobile terminal devices due to its low profile, simple design and ease of integration with other small form factor devices [2]. The different antenna structures are developed with one or more notch bands by using the planar monopole structures [3]. The design of traditional antenna used for broadband applications is suffered by its huge size and limited bandwidths. Moreover, there are various methods are developed to carry the fractal geometry with electromagnetic theory. The modern antenna design namely Minkowski fractal which is used to accomplish the fractal geometry in the antenna design. The usage of fractal antenna simplifies the overall circuit design, minimizes the construction cost and enhances the reliability. Moreover, the fractal antenna doesn't requires any self-loading, extra antenna parts such as capacitors and coils for creating the resonant in the antenna design. The wideband array is obtained in the multiband and amount of elements in the array are 
minimized by organizing the elements in the fractal pattern [4-5].

Accordingly, the fractal shape antennas are considered as an appropriate way for designing the advanced antennas namely multiband antennas. The characteristics of the multiband antennas are similar to the fractal antenna for different frequency bands as well as it has small size antennas [6]. The Ultra-Wide Band (UWB) antenna design uses the fractal geometry due to its space filling and self-similar features. Hence, this UWB antenna obtains the less size and wideband features [7]. The antenna miniaturization or antenna size is minimized by using the space filling property. Moreover, the higher permeability and permittivity of the substrate are also used for minimizing the antenna size [8-9]. There are various antenna structures are already designed, neither it has stable radiation patterns or low-profile structure [10-11]. Since, a high efficient and inexpensive monopole antenna is designed by using the growing printed antenna technology [12]. Additionally, the planar monopole antenna has various physical characteristics such as light weight, less size and broadband. Hence, this monopole antenna is used in portables devices due to its physical characteristics [13-14]. The monopole antenna is designed in different shapes such as ring [15], C-shape [16] and so on. The main contributions of this research paper are given as follows:

- The efficiency of the monopole antenna is improved by using the MMPA design. The monopole antenna is widely used due to its lightweight and less cost.

- The Minkowski Iteration Fractal antenna technique is developed in the MMPA for obtaining the frequency tuning in wideband applications. This fractal antenna provides three different frequencies such as base antenna-3.6 $\mathrm{GHz}$, first iteration-3.4 GHz and second iteration-3.2 GHz.

- Moreover, the return loss, VSWR, electric and magnetic field are analysed for the proposed MMPA design.

The paper is organized as follows, literature review of recent methods in monopole antenna is provided in section 2, the description of monopole microstrip patch antenna is given in section 3, parametric analysis of MMPA is explained in section 4, and comparison of Minkowski monopole with other fractal antenna is discussed in section 5 and conclusion is in section 6 .

\section{Literature review}

In wireless applications, the antennas with the capacity to work on huge frequency band and compact in size have high demand. Recent researches in designing fractal antenna with compact in size are reviewed in this section.

Manohar [17] analysed the super-wideband Koch snowflake antenna for different wireless applications. The Koch iteration techniques of self-similarity and space-filling features has been applied at the triangular patch to acquire the antenna compactness and broadband performance. The proposed antenna has the compact size and stable radiation pattern over entire frequency spectrum. An I-shaped parasitic element was used for stable pattern in this antenna. The experimental result showed that the proposed antenna has a wide-impedance BW without increasing overall dimension. But the return loss and efficiency of the developed antenna were low.

Karmakar [18] developed compact UWB monopole antenna with triple-band, triple-sense circular polarization. The developed design consists of compact quad circular monopole, triangular and rectangular ring resonator, a fractal parasitic ring resonator, and ground plane consists of modified ' $\mathrm{T}$ ' and 'I' shaped slots. The proposed antenna radiates Left-Hand Circular Polarised (LHCP) wave at lower and mid band frequency, and the Right-Hand Circular Polarised (RHCP) wave at upper band frequency. The experimental result showed that the proposed antenna covers an ultra-wide bandwidth and also has good circular polarisation characteristics. The design of the antenna is need to be improved to increase the efficiency of the antenna.

Goswami [19] proposed multi-band planar antenna using a bisected fractal Hilbert Curve. To achieve two additional operating bands apart from the resonant frequency of fractal monopole antenna, two subwavelength Split Ring Resonance (SRR) was placed proximity to the compact monopole. One is Moore shaped fractal SRR, operate at $3.5 \mathrm{GHz}$ and other is conventional SRR, operates at $5.2 \mathrm{GHz}$. A fabricated antenna analysis showed that good agreement between the simulation and experiment. The proposed antenna was compact in size and provide stable monopole-like pattern over the band. The return loss of the monopole antenna was low that affects the overall efficiency.

Elavarasi and Shanmuganantham, [20] designed Koch star fractal antenna with split ring resonator for multi frequency band application. The Circular Split Ring Resonator (CSRR) was fixed on reverse side of the substrate and iterated Koch star. The experimental result showed that the proposed antenna has the 
significant performance in different range. The proposed antenna has the capacity to progress smooth radial model in far field. The antenna design is need to be improved to increases the efficiency.

Singh [21] proposed a multiband circular microstrip patch antenna and analysed on various applications. In order to achieve the desired multiband, a cantor set theory was used to develop fractal slots. The experimental result shows that the projected antenna has the higher performance than the conventional antenna. The proposed antenna is designed on FR4 glass epoxy substrate material and tested using Vector Network Analyzer. The return loss of the proposed antenna is low and its need to be improved. Pokkunuri, Madhav, Sai, Venkateswararao, Ganesh, Tarakaram, and Teja [22] presented the frequency reconfigurable fractal (i.e. Koch Fractal) slot antenna based on the Complimentary Split Ring Resonator (CSRR). This multiband fractal antenna has the snowflake patch as the radiating element which used to develop the Koch Fractal. The frequency reconfigurability was obtained by placing the two PIN diodes in the CSRR. Moreover, this Koch Fractal based multiband antenna was operated in the bands of $1.6 \mathrm{GHz}$ (GPS), $2.4 \mathrm{GHz}$ (Bluetooth) and $5.7 \mathrm{GHz}$ (WiMAX). However, this Koch Fractal antenna had complex ground plane structure that leads to increase the area of the antenna.

\section{Monopole microstrip patch antenna}

The microstrip patch antenna is process at Narrow band frequency and Monopole Microstrip Patch Antenna is process at wide band frequency. The conventional microstrip antenna consists of a metal strip conductor (patch) as an upper layer of a grounded substrate. The factors such as shape, dimension and substrate dielectric constant are determining the microstrip antenna performance in terms of bandwidth and radiation. The rectangular patch monopole antenna with patch size of $20 \times$ $25 \mathrm{~mm}^{2}$ and microstrip feeding line of $3 \times 10 \mathrm{~mm}^{2}$ with rectangular slotted monopole ground of size $30 \times 7 \mathrm{~mm} 2$ is shown in Fig. 1.

This section details about the MPA design process by selecting the essential parameters such as antenna width $(W)$ effective dielectric constant $\left(\varepsilon_{r}^{e f f}\right)$, effective antenna length $\left(L_{e f f}\right)$, length extension $(\Delta L)$, and patch length $(L)$.

\section{- Calculation of antenna width $(W)$}

A practical width $(W)$ is calculated for MPA leads to better radiation efficiency. The mathematical equation to calculate the antenna width is given in the

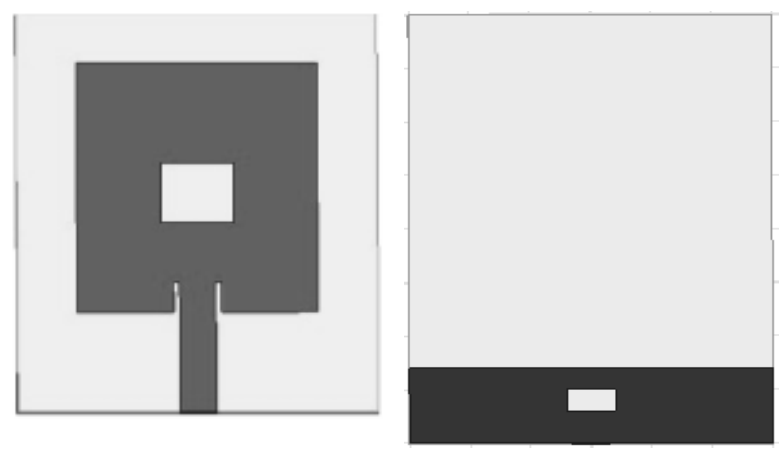

Figure. 1 The structure of rectangular monopole patch antenna

Eq. (1).

$$
W=\frac{c}{2 f_{r}} \sqrt{\frac{2}{\varepsilon_{r}+1}}
$$

Where, $c$ is denoted as velocity of the light $\left(3 \times 10^{8} \mathrm{~m} / \mathrm{s}\right), \varepsilon_{r}$ is stated as relative permittivity $\left(\varepsilon_{r}=3\right)$, and $f_{r}$ is indicated as resonating frequency $\left(f_{r}=2.8 \mathrm{GHZ}\right)$.

\section{- Calculation of effective dielectric constant $\left(\varepsilon_{r}^{e f f}\right)$}

In MPA, the effective dielectric constant $\varepsilon_{r}^{\text {eff }}$ is estimated on the basis of air dielectric boundary as shown in the Eq. (2).

$$
\varepsilon_{r}^{e f f}=\frac{\varepsilon_{r}+1}{2}+\frac{\varepsilon_{r}-1}{2}[1+12(h / W)]^{-1 / 2}
$$

Where, $\varepsilon_{r}=3$.

\section{- Calculation of antenna length $\left(L_{e f f}\right)$}

Generally, the width and length of MPA is calculated by selecting the substrate. The initial approximation of the length is made for a half wave MPA as represented in Eq. (3).

$$
L_{e f f}=\frac{c}{2 \sqrt{\varepsilon_{r}^{e f f}}}\left(\frac{1}{f_{r}}\right)
$$

Where, $f_{r}=2.8 \mathrm{GHZ} \quad, \quad C=3 \times 10^{8} \mathrm{~m} / \mathrm{s}$, and, $\varepsilon_{r}^{\text {eff }}=0.1$.

\section{- Calculation of length extension $(\Delta L)$}

Hence, there is a line extension related with a patch, due to fringing fields and radiating edges of the antenna. The length extension $\Delta L$ is mathematically denoted in the Eq. (4). 


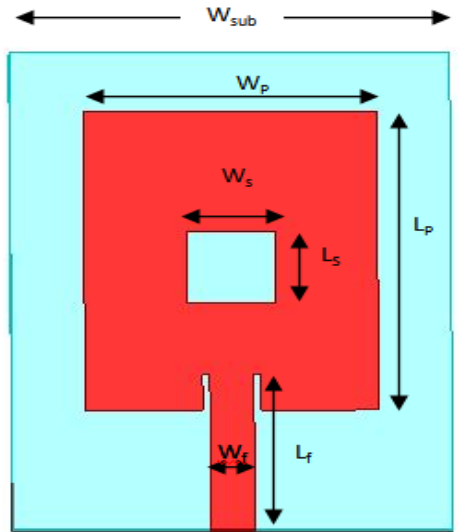

(a)

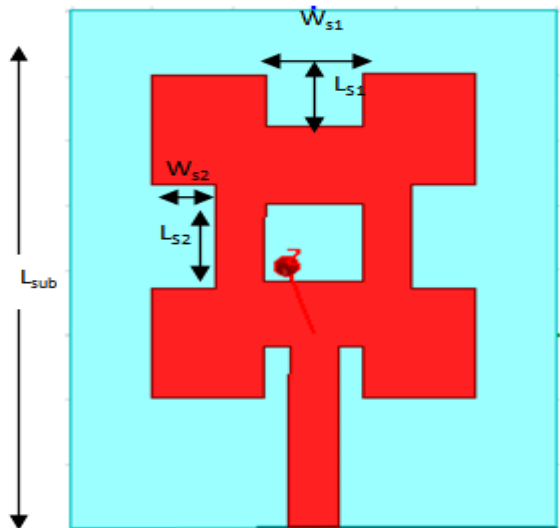

(b)

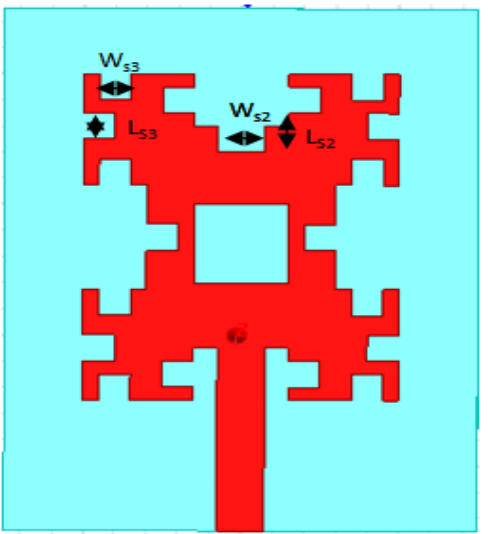

(c)

Figure. 2 Minkowski fractal curve: (a) base structure, (b) first iteration, and (c) second iteration

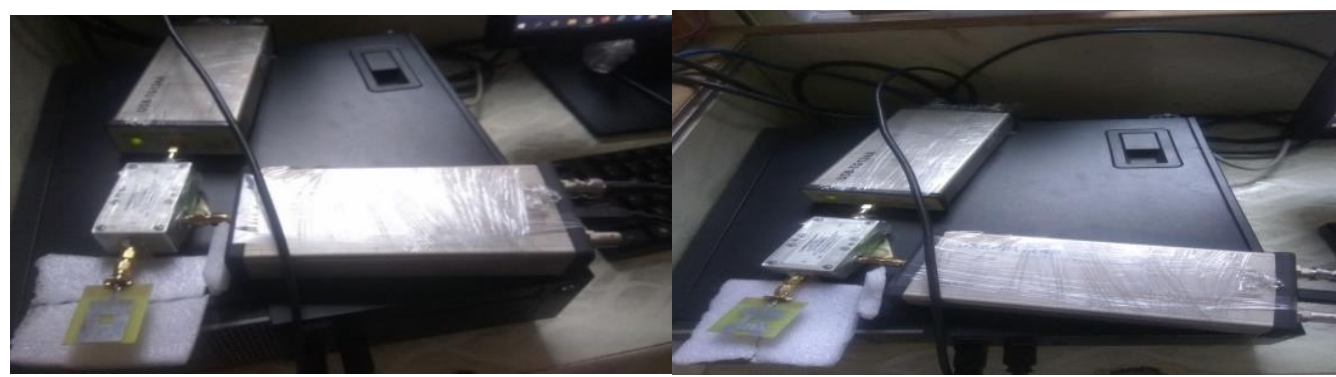

(a)

(b)

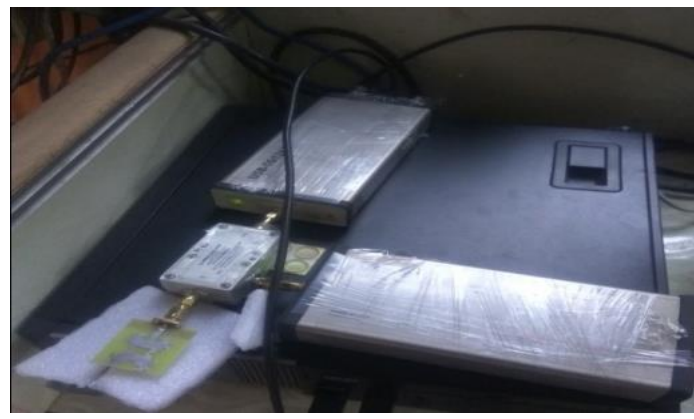

(c)

Figure. 3 Fabricated MMPA antenna: (a) base structure, (b) first iteration, and (c) second iteration

$$
\Delta L=0.412 h\left[\frac{\left(\varepsilon_{r}^{e f f}+0.3\right)\left(\frac{W}{h}+0.264\right)}{\left(\varepsilon_{r}^{e f f}-0.258\right)\left(\frac{W}{h}+0.8\right)}\right]
$$

Where, $\varepsilon_{r}^{\text {eff }}=0.1$.

\section{- Calculation of patch length $(L)$}

Usually, the patch length is determined by using the Eq. (5).

$$
L=L_{e f f}-2 \Delta L
$$

Structure of rectangular patch antenna is graphically indicated in Fig. 1, and the current distribution of MPA at 3.6 GHZ. Although, monopole antenna operates at the Wideband frequency, but return loss of monopole antenna is low that affects the overall efficiency. Therefore, the design of Minkowski fractal iteration antenna is proposed in monopole antenna to improve the return loss.

\subsection{Description of Microstrip Minkowski monopole patch antenna}

The proposed MMPA design is designed under three different iterations. Initially, the dimensions of the Euclidean rectangular MMPA at three different modes are obtained by using the Minkowski slots in MPA. The Minkowski slots and fabricated MMPA design are shown in the Fig. 3.

The width and height of the Substrate, patch, feed and slots of three iterations of MMPA tabulated in Table 1 and are represented in Fig. 2 as Ws, Wp, Wf, Ws1,Ws2 and Ls, Lp, Lf, Ls1, Ls2 respectively and $\epsilon_{r=} 4.4$ is the permittivity and $\mathrm{Hs}=1.6$ is height of the MMPA. 
Table 1. Length and width measurement of proposed

\begin{tabular}{|c|c|}
\hline \multicolumn{1}{|c|}{ MPA } \\
\hline Parameter & Value(mm) \\
\hline Wsub & 30 \\
\hline Lsub & 35 \\
\hline Wp & 20 \\
\hline Lp & 25 \\
\hline Ws & 6 \\
\hline Ls & 6 \\
\hline Wf & 3 \\
\hline Lf & 10 \\
\hline Hs & 1.6 \\
\hline Ws1 & 6 \\
\hline Ls1 & 4 \\
\hline Ws2 & 3 \\
\hline Ls2 & 2 \\
\hline Ws3 & 2 \\
\hline Ls3 & 2 \\
\hline
\end{tabular}

Based on the RDRA design, the microstrip line is used as feed with size of $3 \times 10 \mathrm{~mm}^{2}$ to achieve 50ohm impedance matching. In this MMPA design, the Microstrip feed is selected because of its simple fabrication, less loss and it doesn't requires any drilling of hole. This shows that the Microstrip feed consumes only less space. These points are considered to accomplish the parametric studies for achieving enhanced resonance characteristics. The FR4 with $1.6 \mathrm{~mm}$ height, 4.4 relative permittivity and the dimensions of $30 \mathrm{~mm} \times 40 \mathrm{~mm}$ is selected as substrate for designing the loop. The fractal shape is created by moving the middle one-third of each straight segment (i.e., indentation length) by some fraction namely the indentation width. The Indentation factor is mainly described as the ratio between the indentation width and the indentation length. The resulting architecture has 5 different segments for an each one of the last iteration, but all the 5 segments are not in same scale. The indentation factor variation creates the shift in the resonant frequencies. Therefore an adequate indentation factor's tuning is required for achieving the wideband characteristics.

\section{Parametric analysis of fractal MMPA}

The self-similar properties of fractal geometry are utilized for improving the bandwidth. The Minkowski is considered as good choice for generating the fractal shapes in the monopole antenna. The design restrictions of the Euclidean geometry are selected as the initial parameters of the Fractal MMPA (FMMPA). The parametric study is limited to the $2^{\text {nditeration }}$ by considering the tolerance of fabrication.

\subsection{Rectangular MMPA (Initiator)}

For successive fractal designs, the microstrip fed rectangular MMPA is developed as the initiator. The slots are combined in the feed and patch for achieving the coupling among the feed section and the MMPA as well as this integration is used to obtain the $50 \mathrm{ohm}$ impedance matching. An optimal output from the parametric study are selected as final fabrication design values which are shown in the Table 1 .

\subsection{Indentation width (First iteration)}

The effect of indentation width variation while maintaining the fixed indentation length in one third of the linear segment is explored in the 1st stage of parametric study. The resonant frequency is continuously increased with respect to the increment in indentation factor. The frequency increase is recognized to the reduction in an entire dielectric material. Here, the DRA's resonant frequency is mainly depends on the size or volume of DRA. Therefore, the resonant frequency is minimized, when there is a decrement in the overall volume. The resulting converge is in the wideband due to the higher indentation factor with higher order modes. Moreover, the further increment in the indentation factor maximizes the indentation width that leads to degradation in the impedance matching. This impedance mismatching is occurred due to the unprotected slot loop with higher indentation factor.

\subsection{Indentation width (Second iteration)}

The $2^{\text {nd }}$ order iteration in the RDRA boundary is investigated with the MMPA design. The indentation factor is varied by $3 \times 2 \mathrm{~mm}^{2}$ and $2 \times 2 \mathrm{~mm}^{2}$ and the increase in the indentation factors minimizes the resonant frequencies through the MMPA design. From the fractal DRA's electric field pattern, knows that the $1 \mathrm{st}$ and 2 nd resonances for the fractal geometry are recognized to the bigger inner rectangle of the DRA, whereas the 3rd resonance may recognized to the 4th outer rectangles of the DRA. The integration of fractal geometry provides selfsimilar antenna behaviour which is specified by the connections in the first two resonances. Fig. 4 and Fig. 5 shows the electric-field (E-field) and magnetic field (H-field) distribution of the proposed MMPA design respectively.

The effective dielectric constant $\left(\epsilon_{\text {reff }}\right)$ is derived for identifying the resonant frequency of the DRA, when the fractal geometry is integrated on its boundary. Let, the original dimensions of the RDRA is $A \times B \times H$ and relative permittivity of the RDRA is $\epsilon_{r}$. After completing one iteration, the dimension 


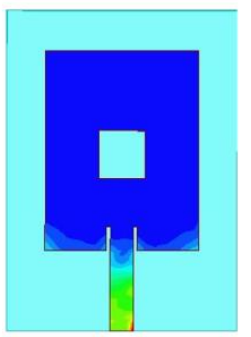

(a)

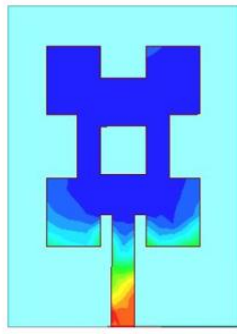

(b)

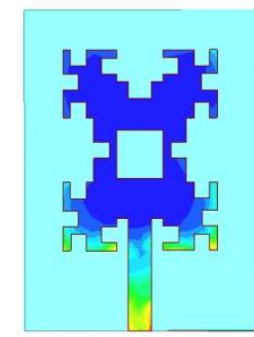

(c)
Figure. 4 Electrical field of design: (a) base design, (b) first iteration, and (c) second iteration

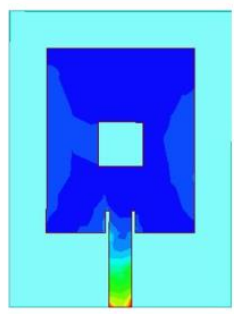

(a)

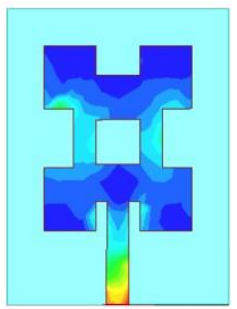

(b)

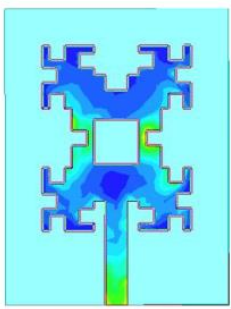

(c)
Figure. 5 Magnetic field of the designs: (a) base design,

(b) first iteration, and (c) second iteration

compromises both the $\epsilon_{0}$ and $\epsilon_{r}$. Therefore, an effective permittivity is allocated to the RDAR for computing the resonant frequency. Moreover, the resonant frequency is calculated by using the Eqs. (6) - (9).

$$
\begin{gathered}
q=2 i \delta^{2} \times \sum_{n=1}^{k} \delta^{n-1} \times\left(\frac{A}{B}+\frac{B}{A}\right) \\
\epsilon_{\text {reff }}=q \times \epsilon_{0}+(1-q) \times \epsilon_{r} \\
k_{x}=\frac{\pi}{A} ; k_{z}=\frac{\pi}{2 H}
\end{gathered}
$$

$$
k_{y} \tan \left(\frac{k_{y} B}{2}\right)=\sqrt{\left(\epsilon_{\text {reff }}-1\right) k_{0}^{2}-k_{y}^{2}}
$$

where, computed filling factor is denoted as $q$ which specifies the dielectric material fraction; scalar factor is denoted as $\delta=(L / 3) / L=1 / 3$; the indentation factor is denoted as $i ; k$ specifies the order of iteration; the length, width and height of the DRA are $A, B$ and $H$ respectively. The relative permittivity of DRA and vacuum are specified as $\epsilon_{r}$ and $\epsilon_{0}$ respectively. Next, the wave numbers in the $x, y$, and $z$ directions are represented as $k_{x}, k_{y}$ and $k_{z}$ respectively. This designed MMPA has different advantages such as bandwidth configuration, frequency reconfigurability and less patch size. The bandwidth configuration and frequency reconfigurability is achieved by designing fractal geometry shape in MMPA which used to obtain three different frequencies such as $3.2 \mathrm{GHz}, 3.4 \mathrm{GHz}$ and 3.6 GHz. Moreover, the area of the MMPA is minimized by designing the patch with less size.

\section{Comparison of minkowski monopole fractal antenna with other fractal antenna}

In this section, the proposed MMPA is compared with other fractal antenna to examine the performance. The return loss of the proposed MMPA and base design antenna is shown in Fig. 6. The return loss of the proposed MMPA design is higher compare to the base design antenna. The return loss of proposed MMPA first iteration design is $-25.66 \mathrm{~dB}$ at $3.4 \mathrm{GHz}$ and the proposed MMPA second iteration has $-21.38 \mathrm{~dB}$ at $3.2 \mathrm{GHz}$. The proposed MMPA structure is compared with one existing antenna namely Koch Fractal antenna [22] to show the efficiency of the MMPA.

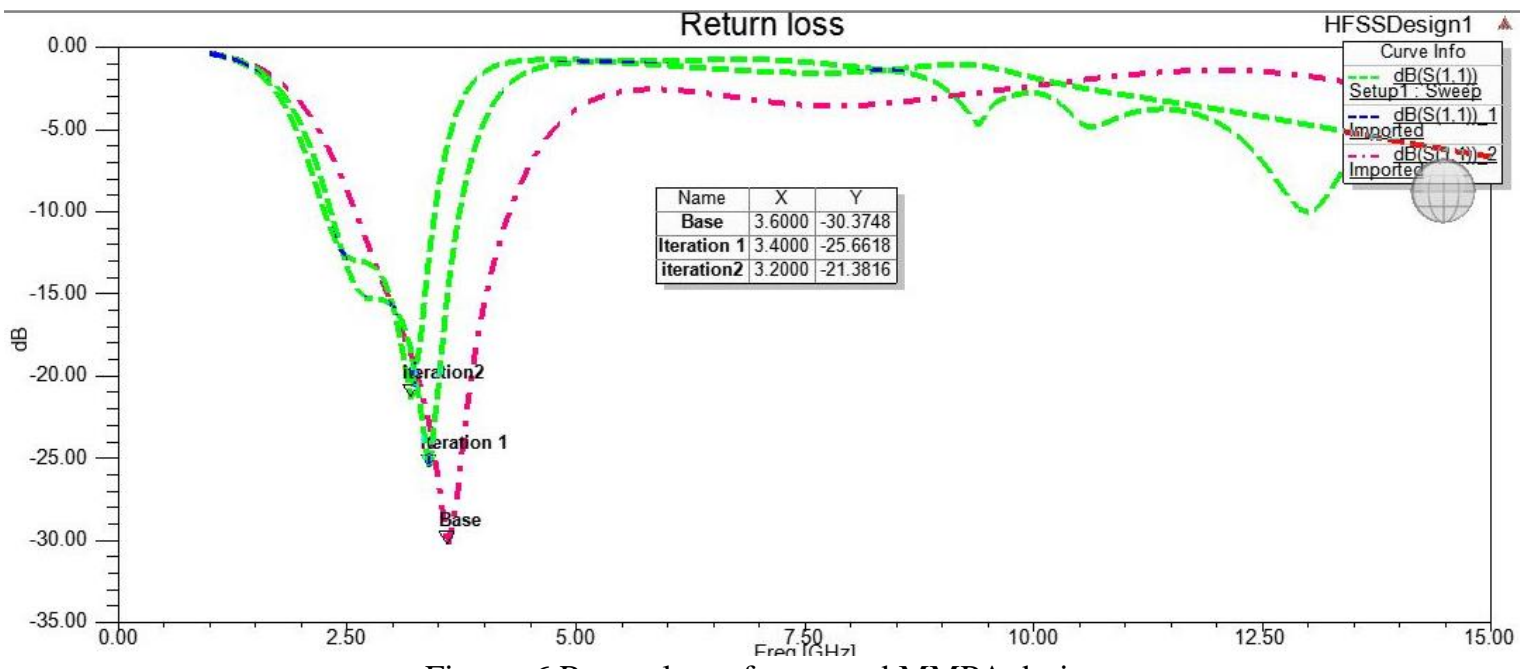

Figure. 6 Return loss of proposed MMPA design 


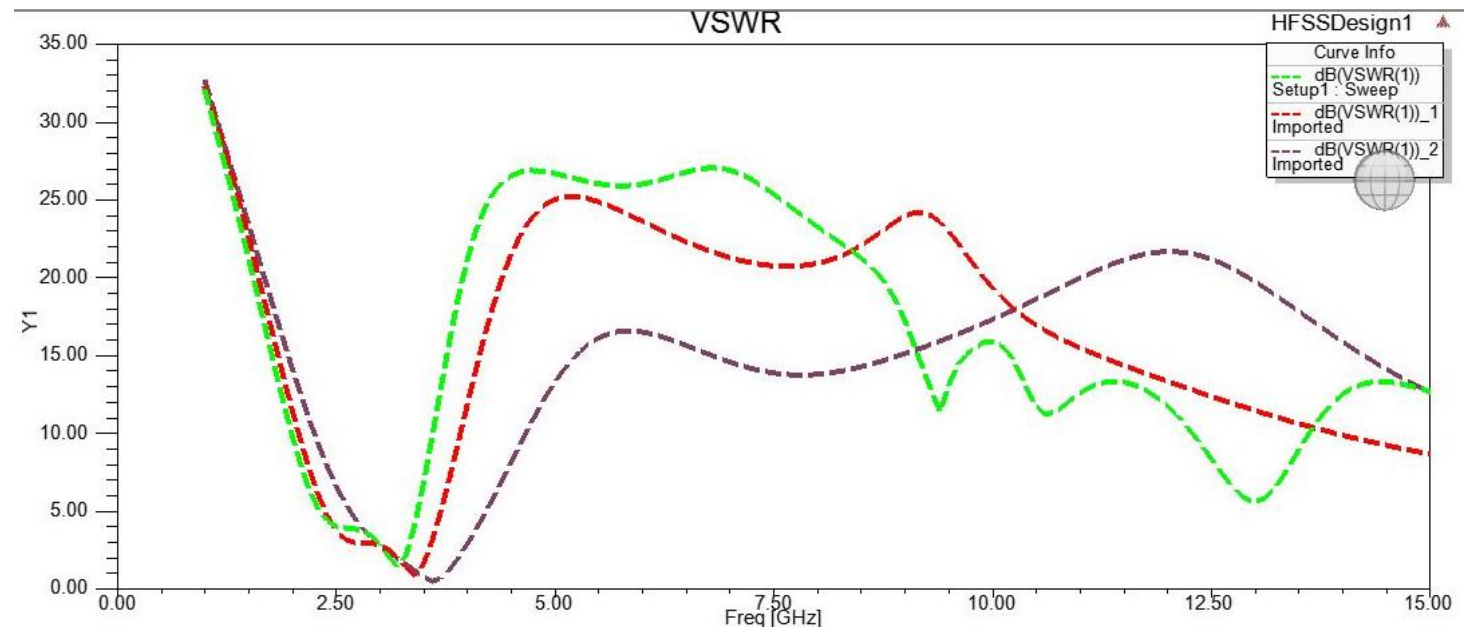

Figure. 7 VSWR of the proposed MMFA design

Table 2. Parameter analysis of proposed MMPA design

\begin{tabular}{|c|c|c|c|c|c|c|}
\hline Name & $\begin{array}{c}\text { Frequency } \\
(\mathbf{G H z})\end{array}$ & Return Loss(dB) & VSWR & Gain & Bandwidth(MHz) & $\begin{array}{c}\text { Area reduction } \\
\text { in \% }\end{array}$ \\
\hline Base & 3.6 & -30.37 & 0.5 & 3.0794 & $2600-4300$ & - \\
\hline First Iteration & 3.4 & -25.66 & 0.9 & 2.8839 & $2300-3700$ & $13 \%$ \\
\hline Second Iteration & 3.2 & -21.38 & 1.2 & 2.5213 & $2300-3452$ & $27 \%$ \\
\hline
\end{tabular}

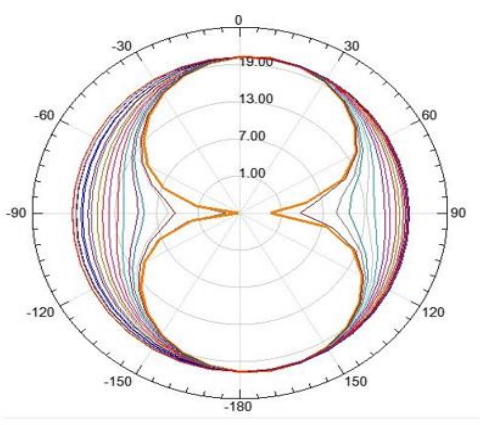

(a)

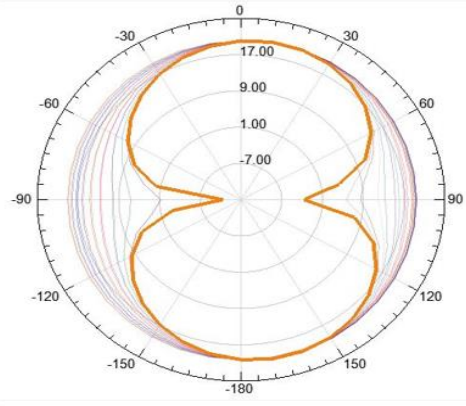

(b)

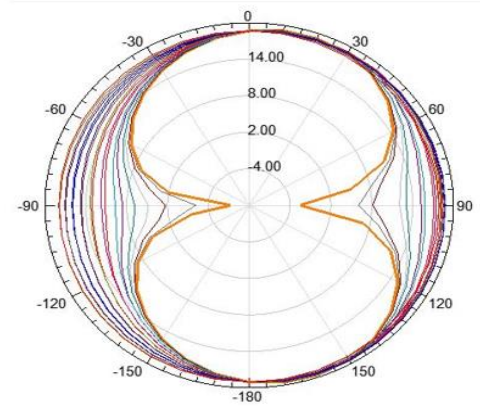

(c)

Figure. 8 Normalized radiation pattern: (a) base design, (b) first iteration, and (c) second iteration

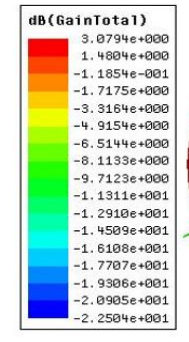

(a)
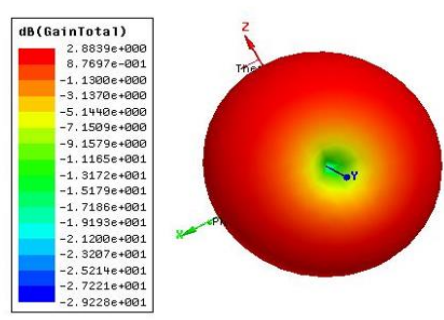

(b)

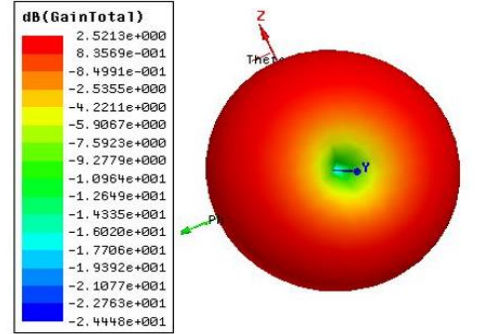

(c)

Figure. 9 Measured antenna gain: (a) base design, (b) first iteration, and (c) second iteration

\subsection{Antenna frequency analysis using fabrication}

The antenna frequency analysis using fabricated MMFA is described in this section. This section analyse the return loss, VSWR, E-field and H-field for the baseband design, first iteration and second iteration. The return loss of the baseband design, first iteration and second iteration are shown in the Fig. 10 (a), 11 (a) and 12 (a) respectively. Similarly, the Fig. 10 (b), 11 (b) and 12 (b) shows the VSWR of the baseband design, first iteration and second iteration respectively. The curve of the return loss Fig. 10 (a), 11 (a) and 12 (a) is used to identify the frequency range of the fractal shape antenna. The curve of Fig. 10 (a), 11 (a) and 12 (a) lies in the $3.6 \mathrm{GHz}, 3.4 \mathrm{GHz}$ and $3.2 \mathrm{GHz}$ of frequency. Moreover the width of 


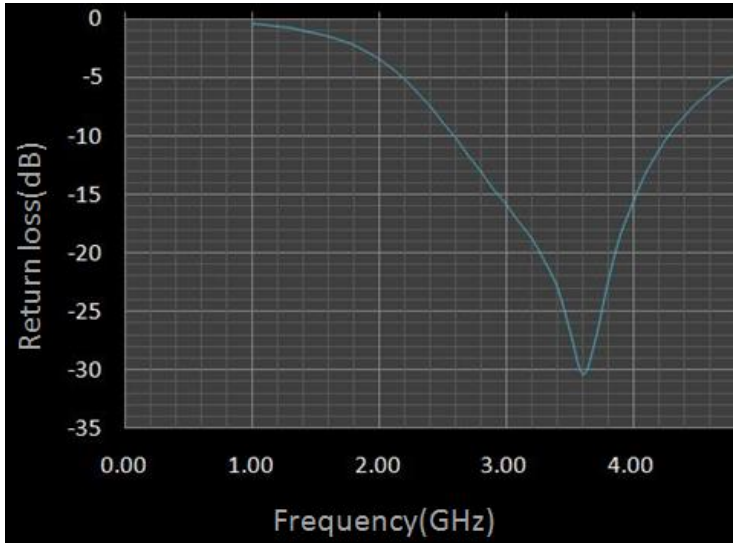

(a)

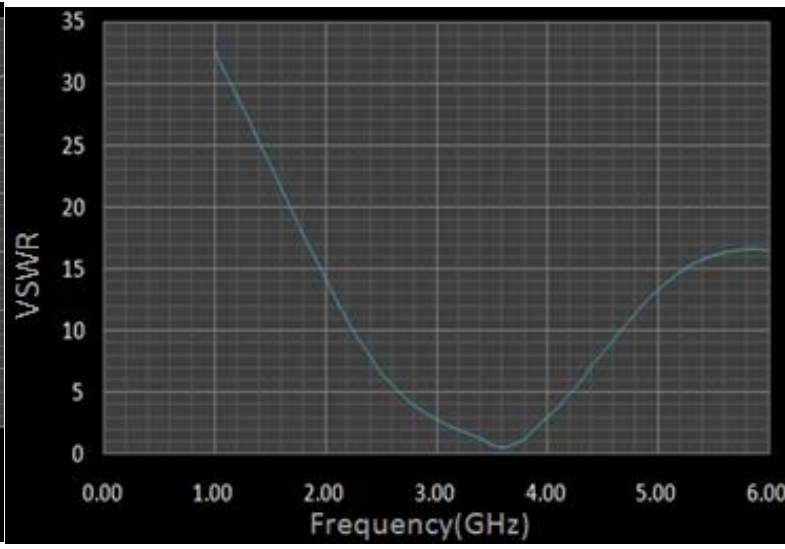

(b)

Figure. 10 Base design: (a) return loss and (b) VSWR

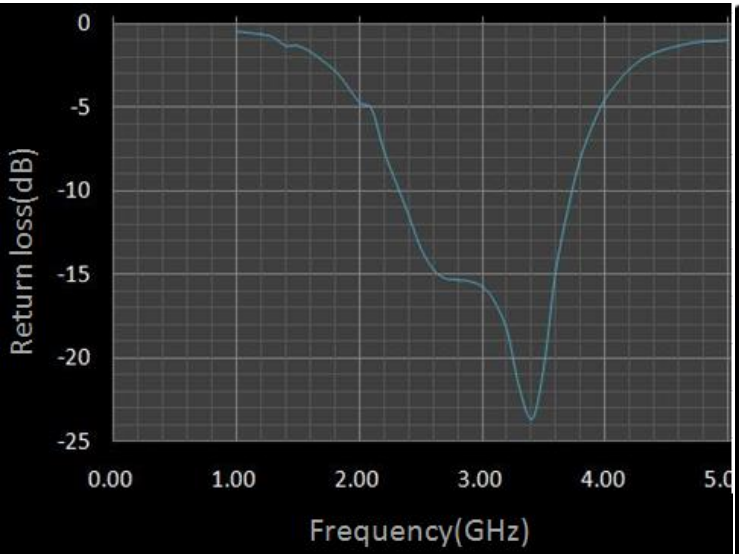

(a)

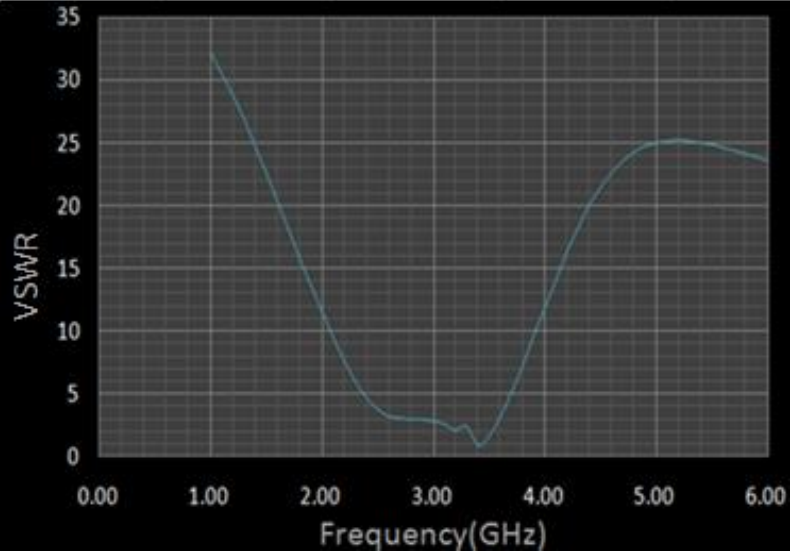

(b)

Figure. 11 First iteration: (a) return loss and (b) VSWR

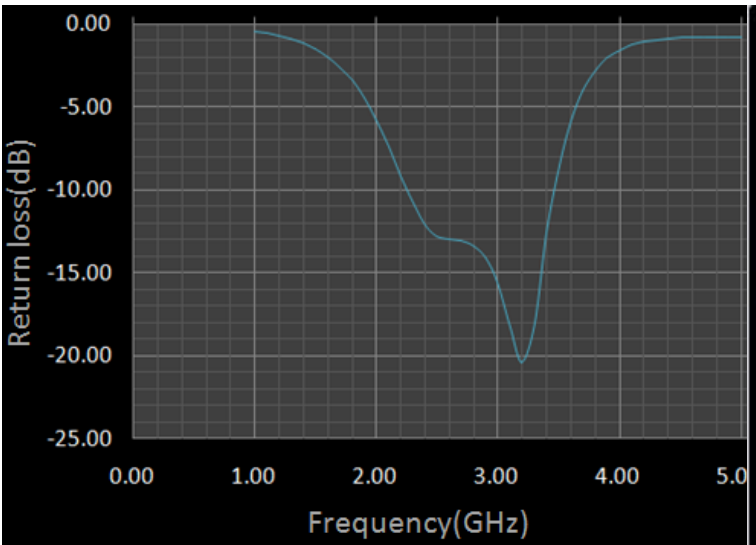

(a)

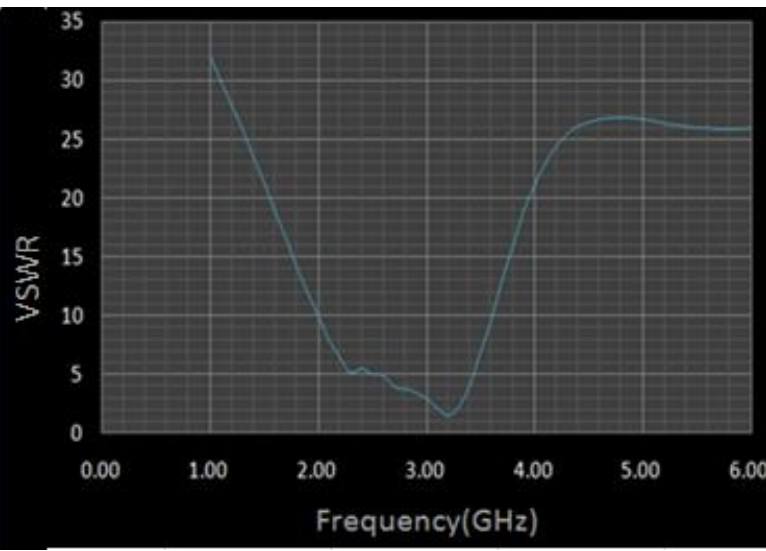

(b)

Figure. 12 Second iteration: (a) Return loss and (b) VSWR

curve at $-10 \mathrm{~dB}$ represents the bandwidth of the MMFA baseband design, first iteration and second iteration.

The radiation pattern for $3.6 \mathrm{GHz}, 3.4 \mathrm{GHz}$ and $3.2 \mathrm{GHz}$ are shown in the Fig. 13, 14 and 15 respectively. In that, Fig. 13.a, 14.a and 15.a shows the E-field radiation patterns of the base case, first iteration and second iteration. Moreover, Fig. 13.b, 14.b and 15.b shows the H-field radiation patterns of the base case, first iteration and second iteration respectively. The MMPA based design is used for improving the efficiency of the monopole antenna as well as the developed antenna has lightweight. The frequency tuning over the wideband applications are obtained by using the minkowski iteration fractal antenna is developed in the MMPA. This MMPA provides three different frequencies such as base antenna-3.6 GHz, first iteration-3.4 


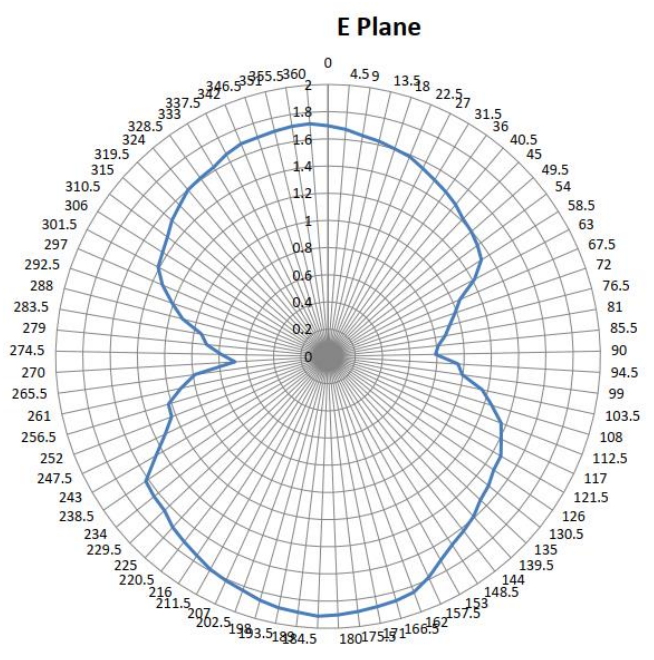

(a)

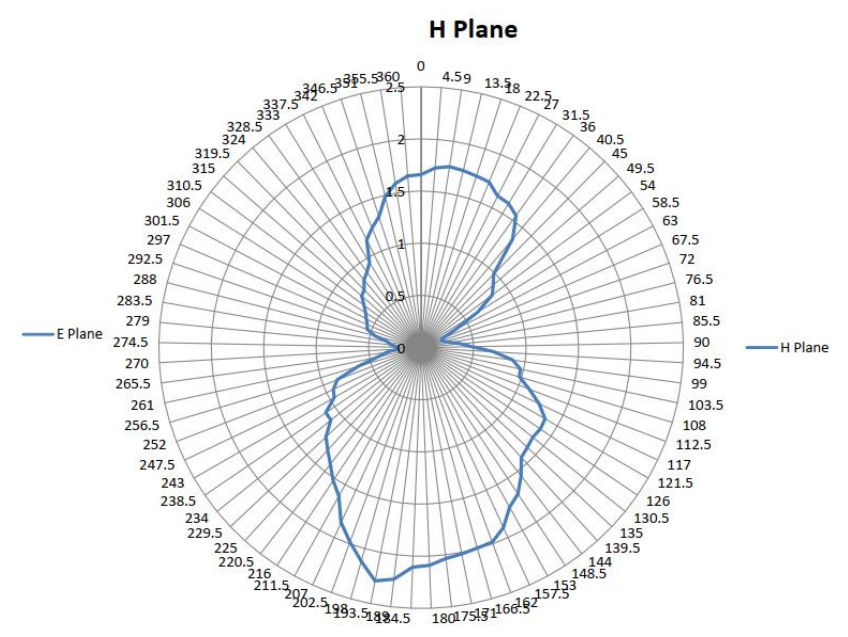

(b)

Figure. 13 Base design: (a) E-fiel, and (b) H-field

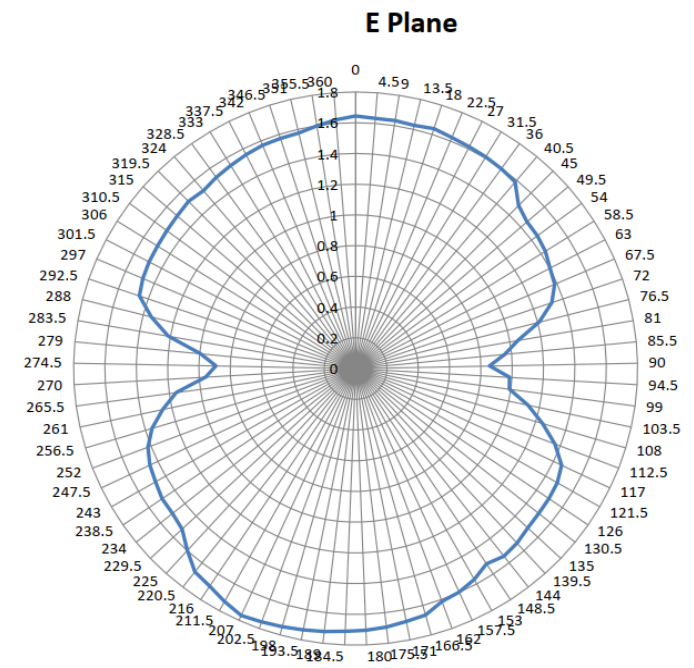

(a)

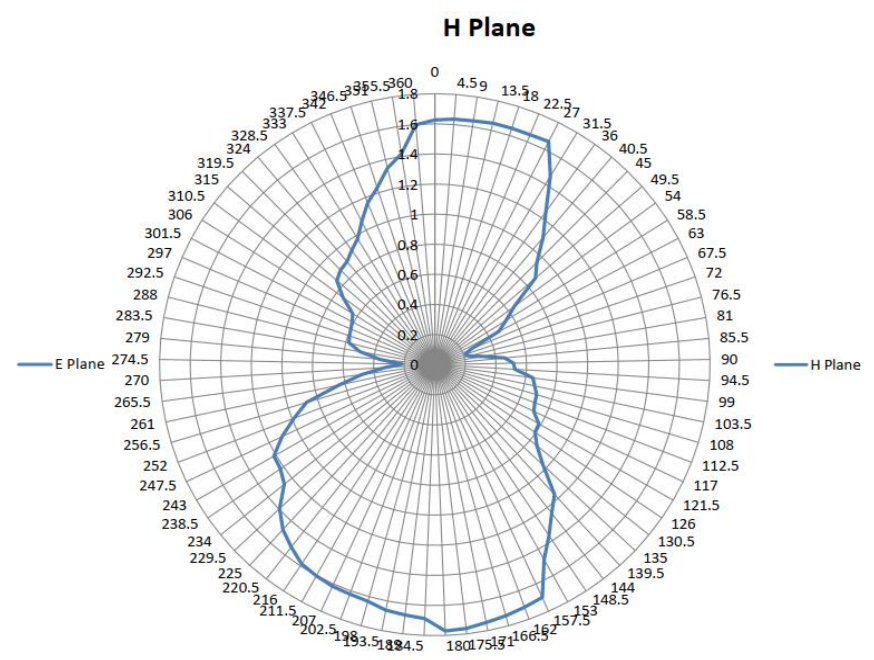

(b)

Figure. 14 First iteration: (a) E-field and (b) H-field
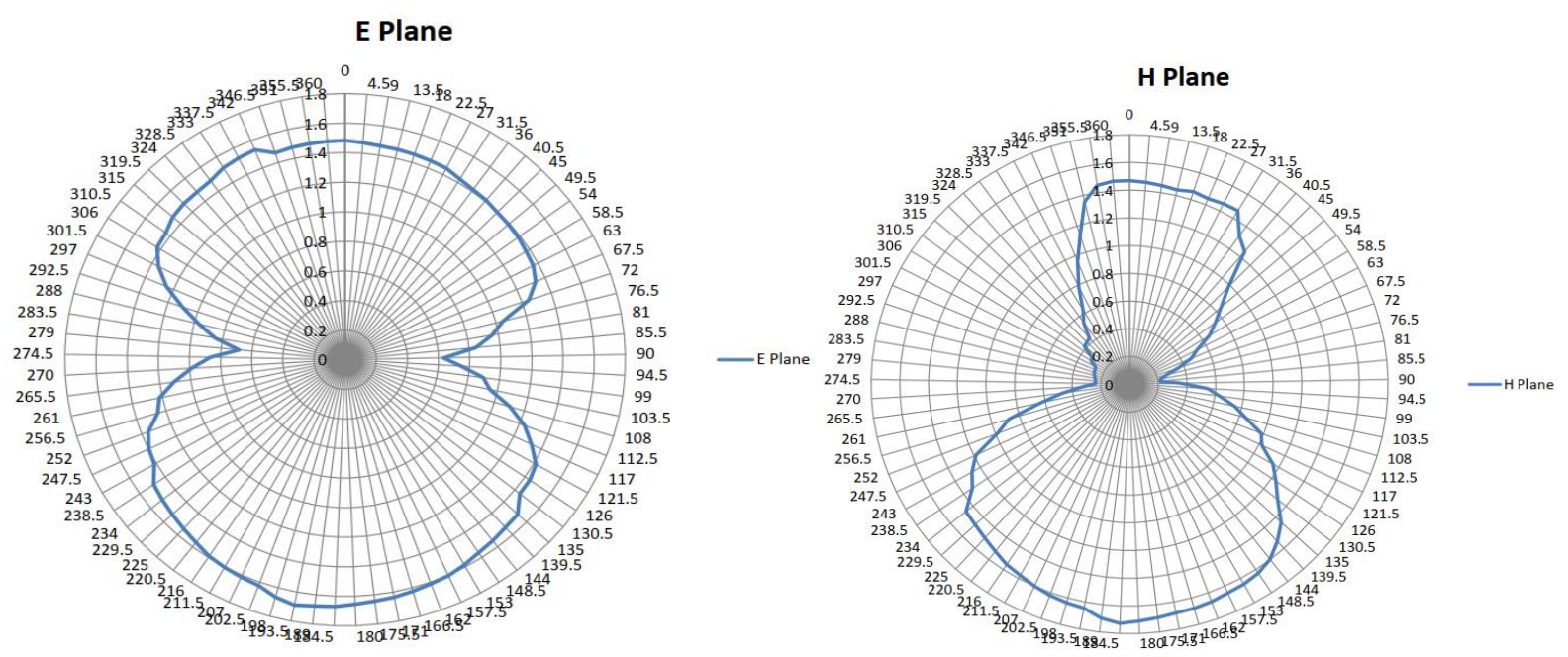

(b)

Figure. 15 Second iteration: (a) E-field and (b) H-field 
Table 3. Comparison of MMPA with Koch Fractal

\begin{tabular}{|c|c|c|}
\hline Parameters & $\begin{array}{c}\text { Koch Fractal } \\
\text { antenna [22] }\end{array}$ & MMPA \\
\hline $\begin{array}{c}\text { Patch dimension } \\
\left(\boldsymbol{m m}^{2}\right)\end{array}$ & $35 \times 30$ & $25 \times 20$ \\
\hline Return loss $(\boldsymbol{d B})$ & -15 to -26 & -21 to -30 \\
\hline
\end{tabular}

$\mathrm{GHz}$ and second iteration-3.2 GHz.

Table 3 shows the comparison of MMPA with Koch fractal antenna [22] in terms of patch dimension and return loss. From the Table 3, knows that the MMPA has lesser patch dimension and return loss than the Koch Fractal antenna [22]. The ground plane of the Koch Fractal antenna [22] has complex structure whereas the proposed MMPA design is designed only by using rectangular shape with $7 \mathrm{~mm}$ of size that used to minimize the area of MMPA. This rectangular shape based ground plane is used to minimize the cost while designing the MMPA. The snowflake structure increases the area of the overall Koch Fractal antenna [22] by adding extra triangle shape antenna in iteration 1. Moreover, the Koch Fractal antenna [22] uses metamaterial for minimizing the return loss. But, the MMPA obtains less return loss in the range of $-21 \mathrm{~dB}$ to $-30 \mathrm{~dB}$ without any metamaterial structure. Therefore, the designed MMPA provides different advantages such as bandwidth configuration, frequency reconfigurability and less patch size. This MMPA provides three different frequencies such as $3.2 \mathrm{GHz}$, $3.4 \mathrm{GHz}$ and $3.6 \mathrm{GHz}$ in second iteration, first iteration and base design respectively.

\section{Conclusion}

Monopole antenna supports wideband application and also smaller in size, suitable for the wireless communication application. Monopole antenna has the limitation of lower return loss that affects the efficiency of the antenna. In this research, MMPA design technique is proposed to improve the return loss and efficiency. The Minkowski Fractal Iteration technique is applied in the monopole antenna. The experimental result shows that the developed antenna design has the higher efficiency compared to other fractal and monopole antennas. The return loss of the proposed MMPA first iteration is $-25.66 \mathrm{~dB}$ at $3.4 \mathrm{GHz}$ and base design has -30.37 $\mathrm{dB}$ at $3.6 \mathrm{GHz}$. The patch dimension of the MMPA is $25 \times 20 \mathrm{~mm}^{2}$, it is less when compared to the Koch fractal antenna. The antenna fractal design is improved in future work to enhance the bandwidth efficiency.

\section{Conflicts of Interest}

The authors declare no conflict of interest.

\section{Author Contributions}

The paper conceptualization, methodology, software, writing - original draft preparation, writing - review and editing, visualization, have been done by $1^{\text {st }}$ author. The supervision and project administration, have been done by $2^{\text {nd }}$ author. Validation, formal analysis, investigation, resources, data curation is done by $3^{\text {rd }}$ author.

\section{Acknowledgments}

Acknowledgments are to show that the article is supported by what organization. For example, "This work was supported by the National Nature Science Foundation under Grant No. 405".

\section{References}

[1] S. Dhar, R. Ghatak, B. Gupta, and D. R. S. Poddar, "A Wideband Minkowski Fractal Dielectric Resonator Antenna", IEEE Transactions on Antennas and Propagation, Vol. 61, No. 6, pp. 2895- 2903, 2013.

[2] S. Verma and P. Kumar, "Printed multiband Minkowski fractal curved antenna", In: Proc. of Twentieth National Conf. on Communications (NCC), pp. 1-5, 2014.

[3] B. T. P. Madhav, M. V. Rao, and T. Anilkumar, "Conformal band notched circular monopole antenna loaded with split ring resonator", Wireless Personal Communications, Vol. 103, No. 3, pp. 1965-1976, 2018.

[4] I. T. Sesiaand and M. Baker, "LTE - The UMTS Long Term Evolution: From Theory to Practice", Chichester, U. K.: Wiley. 2009.

[5] M. K. Meshram, R. K. Animeh Pimpale, and N. K. Nikolova, "A Novel Quad-band Diversity Antenna for LTE and Wi-Fi Applications with High Isolation", IEEE Trans. Antennas Propag., Vol. 60, No. 9, pp. 4360-4371, 2012.

[6] X. Zhou, X. L. Quan, and L. Ronglin, "A Dual Broadband MIMO Antenna System for GSM/UMTS/LTE and WLAN Handsets", IEEE Antennas Wireless Propag., Vol. 11, pp. 551-554, 2012.

[7] S. Tripathi, A. Mohan, and S. Yadav, "A compact UWB Koch fractal antenna for UWB antenna Array applications", Wireless Personal Communications, Vol. 92, No. 4, pp. 1423-1442, 2017. 
[8] J. S. Sivia, G. Kaur, and A. K. Sarao, “A modified sierpinski carpet fractal antenna for multiband applications", Wireless Personal Communications, Vol. 95, No. 4, pp. 4269-4279, 2017.

[9] S. S. Bhatia, J. S. Sivia, and N. Sharma, "An optimal design of fractal antenna with modified ground structure for wideband applications", Wireless Personal Communications, Vol. 103, No. 3, pp. 1977-1991.

[10] Y. K. Choukiker and S. K. Behera, "Wideband frequency reconfigurable Koch snowflake fractal antenna", IET Microwaves, Antennas and Propagation, Vol. 11, No. 2, pp. 203-208, 2017.

[11] S. Jindal, J. S. Siviaand H. S. Bindra, "Hybrid fractal antenna using meander and Minkowski curves for wireless applications", Wireless Personal Communications, Vol. 109, No. 3, pp. 1471-1490.

[12] K. P. Ray and S. S. Thakur, "Modified trident UWB printed monopole antenna", Wireless Personal Communications, Vol. 109, No. 3, pp. 1689-1697, 2019.

[13] T. H. Kim, Y. Kim, T. H. Yoo, and J. G. Yook, "Wideband planar monopole antenna for digital TV reception and UHF band communications", IET Microwaves, Antennas and Propagation, Vol. 12, No. 13, pp. 2041-2045, 2018.

[14] Z. X. Xia, K. W. Leung, M. W. Lee, and N. Yang, "Miniature dual-band meander-line monopole chip antenna with independent band control", IEEE Antennas and Wireless Propagation Letters, Vol. 18, No. 9, pp. 18731877, 2019.

[15] R. S. Daniel, R. Pandeeswari, and S. Raghavan, "Dual-band monopole antenna loaded with ELC metamaterial resonator for WiMAX and WLAN applications", Applied Physics A, Vol. 124, No. 8, pp. 570, 2018.

[16] H. H. Tran, N. Nguyen-Trong, and A. M. Abbosh, "Simple design procedure of a broadband circularly polarized slot monopole antenna assisted by characteristic mode analysis", IEEE Access, Vol. 6, pp. 78386-78393, 2018.

[17] M. Manohar, "Miniaturised low-profile superwideband Koch snowflake fractal monopole slot antenna with improved BW and stabilised radiation pattern", IET Microwaves, Antennas and Propagation, Vol. 13, No. 11, pp. 1948-1954, 2019.

[18] A. Karmakar, P. Chakraborty, U. Banerjee, and A. Saha, "Combined triple band circularly polarised and compact UWB monopole antenna",
IET Microwaves, Antennas and Propagation, Vol. 13, No. 9, pp. 1306-1311, 2019.

[19] C. Goswami, R. Ghatak, and D. R. Poddar, "Multi-band bisected Hilbert monopole antenna loaded with multiple subwavelength split-ring resonators", IET Microwaves, Antennas and Propagation, Vol. 12, No. 10, pp. 1719-1727, 2018.

[20] C. Elavarasi, and T. Shanmuganantham, "Multiband SRR loaded Koch star fractal antenna", Alexandria Engineering Journal, Vol. 57, No. 3, pp. 1549-1555, 2018.

[21] S. J. Singh, G. Singh, and G. Bharti, "Circular microstrip antenna with fractal slots for multiband applications", Journal of The Institution of Engineers (India): Series B, Vol. 98, No. 5, pp. 441-447, 2017.

[22] P. Pokkunuri, B. T. P. Madhav, G. K. Sai, M. Venkateswararao, B. Ganesh, N. Tarakaram, and D. Teja, "Metamaterial Inspired Reconfigurable Fractal Monopole Antenna for Multi-band Applications", Int. J. Intell. Eng. Syst, Vol. 12, pp. 53-61, 2019. 